\section{CRISPR in the liver}

\section{By Amy Donner, Senior Editor}

Despite the fact that the therapeutic utility of CRISPR-based approaches has yet to be demonstrated, venture dollars keep flowing into new companies developing the platform. But proof of concept may come faster than expected as new findings show that a CRIPSR-based compound can correct a mutation in adult mice with genetic liver disease. ${ }^{1}$

Last year, multiple independent teams adapted a newly identified bacterial defense system to create a platform for site-directed genome editing. ${ }^{2-7}$ The platform relies on a synthetic single guide RNA (sgRNA) to target the activity of the CRISPR (clustered, regularly interspaced short palindromic repeats)-associated bacterial endonuclease Cas9-the system is referred to in short as CRISPR-Cas9.

Cas9 introduces a double-strand break in genomic DNA in a location designated by complementary interactions between the sgRNA and the DNA target, which triggers DNA repair and genome editing.

Because editing specificity is driven by the sequence of the sgRNA, the CRISPR-Cas9 system is simpler and cheaper than other genomeediting tools, such as zinc finger nucleases (ZFNs) and transcription activator-like effector nucleases (TALENs), which use engineered, DNA-binding proteins to site-specifically target DNA.

CRISPR-Cas9 has been applied in embryos to engineer mouse and nonhuman primate models of human disease ${ }^{8,9}$ The platform also has been used to engineer complex mouse models by simultaneously introducing multiple gene mutations into embryonic stem cells. ${ }^{10,11}$

The first foray into therapeutic application occurred last year, when Editas Medicine was founded to use CRISPR-Cas9 to correct genomic defects in undisclosed diseases. Editas raised $\$ 43$ million and brought together 5 scientific cofounders who spearheaded the conversion of the bacterial defense system to a programmable genome-editing tool.

Last week, the first CRISPR-Cas9 patent for genome-editing applications was assigned to the Broad Institute of MIT and Harvard and the Massachusetts Institute of Technology (MIT). The patent covers CRISPR-Cas9 systems engineered to work in eukaryotic cells, especially human cells, and methods of using these systems. For research use, the technology will be available to anyone on a nonexclusive basis. The Broad Institute did not disclose further licensing details.

Today, CRISPR Therapeutics, which aims to translate CRISPRCas9 into medicines with the potential to cure human genetic diseases, emerged from stealth mode. CRISPR Therapeutics was founded by Versant Ventures and raised $\$ 25$ million.

Like Editas, CRISPR Therapeutics has not yet disclosed the specific targets or indications it will pursue.

CRISPR Therapeutics' scientific founders are Emmanuelle Charpentier, Daniel Anderson, Matthew Porteus, Chad Cowan and Craig Mello.
Charpentier is a professor at the Laboratory for Molecular Infection Medicine at Umeå University, the Helmholtz Centre for Infection Research and Hannover Medical School.

Anderson is an associate professor of chemical engineering and medical engineering and science at MIT and an intramural member of The David H. Koch Institute for Integrative Cancer Research at MIT.

Porteus is an associate professor of pediatrics at the Stanford University School of Medicine, and Cowan is an associate professor of stem cell and regenerative biology at Harvard University and Massachusetts General Hospital.

Mello is chair of molecular medicine and co-director of the RNA Therapeutics Institute at the University of Massachusetts Medical School and a Howard Hughes Medical Institute (HHMI) investigator.

\section{Crisp solution}

Anderson and his team have now for the first time used CRISPR-Cas9 therapeutically. The researchers chose tyrosinemia type I for their proofof-concept studies.

Tyrosinemia type I is a genetic disorder caused by deficiency in fumarylacetoacetate hydrolase (FAH), an enzyme required to break down the amino acid tyrosine. In the absence of this enzyme activity, toxic metabolites accumulate and cause liver or kidney failure. The disease affects about 1 in 100,000 people worldwide.

According to Hao Yin, a postdoctoral associate at the David $\mathrm{H}$. Koch Institute and one of two lead authors on the study, because the disease is the direct result of the presence of an aberrant version of only one gene, using a mouse model of the disease containing a mutated version of the gene offered a straightforward way to establish proof of concept. "The phenotype mimics the human situation. It is caused by a point mutation, and correction can produce stable protein and stable mRNA, which are easy to detect even if editing occurs only in a small portion of cells," said Yin.

The team's CRISPR-Cas9 therapeutic was composed of a vector expressing a Fah-targeting sgRNA and Cas 9 along with a second molecule-the Fah-correcting donor DNA. Tail vein injection of the therapeutic prevented weight loss, whereas mutant mice receiving saline, Fah-correcting DNA alone or empty vector alone rapidly lost $20 \%$ of their body weight.

The therapeutic decreased liver toxicity as demonstrated by decreased liver enzyme activity compared with controls and improved liver histology. The CRISPR-Cas9 therapeutic also increased the amount of spliced Fah mRNA to up to $36 \%$ of wild-type levels and yielded $\mathrm{Fah}^{+}$hepatocytes.

The authors estimated that the therapeutic corrected the Fah gene mutation in about 1 in 250 liver cells, which was sufficient to alleviate disease symptoms.

"It is important to consider whether you have to correct every cell or just a small percentage of cells. For tyrosine metabolism, you don't need the protein made in all the cells, so in this indication, a fractional correction is sufficient," said Art Krieg, SVP and CSO of Sarepta Therapeutics Inc. He formerly was CEO of RaNA Therapeutics Inc., CSO of Pfizer Inc.'s Oligonucleotide Therapeutics Unit and has more than 20 years of experience with oligonucleotide-based therapies. 
Off-site editing activity ${ }^{12-15}$ was not a problem in the study. In cultured cells, genome editing did not lead to detectable cutting at computationally predicted off-target sites.

The therapeutic was well tolerated. After three months, the treated mice had stable body weight, no signs of hyperplasia in the liver and no detectable tumors, which could be induced by off-target editing.

Data were published in

"The paper is very important with regard to in vivo applicability."

$$
\begin{array}{r}
\text {-Rodger Novak, } \\
\text { CRISPR Therapeutics }
\end{array}
$$

Nature Biotechnology. The team also included researchers from Oregon Health \& Science University and the Skolkovo Institute of Science and Technology.

"We believe that showing that the CRISPR-Cas9 system can correct a disease mutation and phenotype in vivo is the first step to turn it into therapy," said Anderson. "These observations on the CRISPR-Cas9 system, in combination with recent advances in nucleic acid delivery technology, make us optimistic that this technology can be used therapeutically."

"The paper is very important with regard to in vivo applicability," said Rodger Novak, CEO of CRISPR Therapeutics.

"The principle of being able to achieve delivery and a level of editing that was efficient and accurate enough to lead to phenotypic disease correction is mind blowing," said Jennifer Doudna. "Even a month ago, we might not have imagined that this kind of application was possible. The discovery of this platform technology was reported in the scientific literature less than two years ago."

Doudna is a professor of molecular and cell biology and chemistry at the University of California, Berkeley, a faculty affiliate of physical biosciences of the Lawrence Berkeley National Laboratory and an HHMI investigator. She also is a scientific cofounder of Editas.

\section{Special delivery}

Delivery and off-target activity are still the primary challenges for any CRISPR-Cas9-based therapeutics. Indeed, the authors of the new paper acknowledged that they stacked the deck in their favorchoosing a disease of the liver, in which delivery is less of a challenge and high-efficiency editing is not necessary to alter the disease.

"Hydrodynamic tail vein injection is great for delivery in mice, but it is not clinically relevant," noted Krieg.

Novak agreed that delivery is a challenge that remains to be overcome. He noted that in the study, hepatocytes with the gene correction expanded and repopulated the liver at the expense of hepatocytes with the mutation. ${ }^{16}$ He said that the selective pressure that increased the number of healthy cells mitigated the low editing efficiency.

In other indications and in other tissues, the selective advantage will not be there to help, he added.

The authors are planning to address delivery. Yin expects that the efficiency of targeting in hepatocytes can be improved with formulations that better allow delivery of nucleic acids to liver, including adeno-associated viruses or nanoparticles.

Another issue Krieg pinpointed was that CRISPR-Cas9 systems carry a greater risk of off-target activity than other oligonucleotidebased platforms.

But Novak expects that off-target concerns will be manageable.
"The sgRNA is critical in determining the amount and effect of offtarget activity. For some sgRNAs, with the most sensitive methods available we don't see any off-target activity and with some you see only a few sites. The design of the sgRNA is extremely simple, so we can choose the ones with the best activity and with a beneficial ratio of on-off target activity."

He said that the two important considerations for off-target activity are the number of off-target sites and the location of those sites.

"For a good sgRNA, yielding only two to three additional doublestranded breaks somewhere in the genome, we need to determine what kind of genetic elements are impacted. More research is needed to understand this," said Novak.

Rachel Haurwitz, president and CEO of Caribou Biosciences Inc. added, "It will be very important for the field to develop unbiased methods to evaluate off-target cleavage that do not rely on computational predictions."

Caribou was founded in 2011 and is using CRISPR-Cas9 technology to develop R\&D tools.

\section{The therapeutic road}

According to Krieg, pursuing ex vivo applications with the editing platform looks to be the best strategy for the short term.

Doudna also likes ex vivo applications because it derisks the platform. "You can ensure correct targeting before putting the cells back into the patient," she said.

Otherwise, she saw multiple paths forward. "People will try to target the liver and maybe the blood, where you can do the targeting ex vivo. Another attractive target is the eye,
"It will be very important for the field to develop unbiased methods to evaluate offtarget cleavage that do not rely on computational predictions." Caribou Biosciences Inc.
-Rachel Haurwitz, where delivery options such as injection can be more efficient than for other tissue types."

For nucleic acid therapeutics, "there are many ex vivo applications that one could consider, and CRISPR-Cas9 could be promising for ex vivo," said John Maraganore, CEO of Alnylam Pharmaceuticals Inc., which develops RNAi therapeutics.

Alnylam is focused on in vivo applications of RNA therapeutics to the liver. "It's not that we can't get delivery to other cell types and tissues, but with the liver we have a very robust set of preclinical and now clinical data that give us great confidence that we can build our pipeline. The liver is going to keep us busy for a very long time," said Maraganore.

Feng Zhang, a scientific cofounder of Editas, would not discuss the company's plans but said that in general CRISPR therapies should be best suited to diseases like HIV, in which turning down gene expression is desirable, and diseases like sickle cell or hemophilia, in which repairing a mutation is the goal.

Zhang is a core member at the Broad Institute and an investigator at the McGovern Institute for Brain Research at MIT.

CRISPR Therapeutics will primarily focus on ex vivo therapeutic applications. "We know how to deliver into hematopoietic stem cells with high efficiency," Novak said. He did not disclose more specific plans but estimated that an IND submission is about two to three years 


\section{ANALYSIS}

away. CRISPR Therapeutics also will develop delivery systems so it can eventually pursue in vivo applications.

The study's findings are patented, and discussions with pharmaceutical companies for licensing and partnering are ongoing.

Donner, A. SciBX 7(16); doi:10.1038/scibx.2014.447

Published online April 24, 2014

\section{REFERENCES}

1. Yin, H. et al. Nat. Biotechnol.; published online March 30, 2014; doi:10.1038/nbt.2884

Contact: Daniel G. Anderson, Massachusetts Institute of Technology, Cambridge, Mass.

e-mail: dgander@mit.edu

2. Mali, P. et al. Science $339,823-826$ (2013)

3. Cong, L. et al. Science 339, 819-823 (2013)

4. Cho, S.W. et al. Nat. Biotechnol. 31, 230-232 (2013)

5. Hwang, W.Y. et al. Nat. Biotechnol. 31, 227-229 (2013)

6. Jiang, W. et al. Nat. Biotechnol. 31, 233-239 (2013)

7. Jinek, M. et al. eLife 2, e00471; published online Jan. 29, 2013; doi:10.7552/eLife.00471

8. Wang, H. et al. Cell 153, 910-918 (2013)

9. Niu, Y. et al. Cell 156, 836-843 (2014)

10. Yang, H. et al. Cell 154, 1370-1379 (2013)

11. Cain, C. SciBX 6(19); doi:10.1038/scibx.2013.455

12. Cain, C. SciBX 6(4); doi:10.1038/scibx.2013.77

13. Fu, Y. et al. Nat. Biotechnol. 31, 822-826 (2013)

14. Hsu, P.D. et al. Nat. Biotechnol. 31, 827-832 (2013)

15. Mali, P. et al. Nat. Biotechnol. 31, 833-838 (2013)

16. Paulk, N.K. et al. Hepatology 51, 1200-1208 (2010)
COMPANIES AND INSTITUTIONS MENTIONED

Alnylam Pharmaceuticals Inc. (NASDAQ: ALNY),

Cambridge, Mass.

Broad Institute of MIT and Harvard, Cambridge, Mass.

Caribou Biosciences Inc., Berkeley Calif.

CRISPR Therapeutics, Basel, Switzerland

The David H. Koch Institute for Integrative Cancer Research at MIT, Cambridge, Mass.

Editas Medicine, Cambridge, Mass.

Hannover Medical School, Hannover, Germany

Harvard University, Cambridge, Mass.

Helmholtz Centre for Infection Research,

Braunschweig, Germany

Howard Hughes Medical Institute, Chevy Chase, Md.

Lawrence Berkeley National Laboratory, Berkeley, Calif.

Massachusetts General Hospital, Boston, Mass.

Massachusetts Institute of Technology, Cambridge, Mass.

McGovern Institute for Brain Research at MIT,

Cambridge, Mass.

Oregon Health \& Science University, Beaverton, Ore.

Pfizer Inc. (NYSE:PFE), New York, N.Y.

RaNA Therapeutics Inc., Cambridge, Mass.

Sarepta Therapeutics Inc. (NASDAQ:SRPT), Cambridge, Mass.

Skolkovo Institute of Science and Technology, Skolkovo, Russia

Stanford University School of Medicine, Stanford, Calif.

Umeå University, Umeå, Sweden

University of California, Berkeley, Calif.

University of Massachusetts Medical School,

Worcester, Mass.

Versant Ventures, San Francisco, Calif. 